\title{
Hydrodynamical simulations of protoplanetary disks including irradiation of stellar photons
}

\section{Resolution study for vertical shear instability}

\author{
Lizxandra Flores-Rivera ${ }^{1}$, Mario Flock ${ }^{1}$, and Riouhei Nakatani ${ }^{2}$
}

\author{
${ }^{1}$ Max-Planck Institute for Astronomy, Königstuhl 17, 69117 Heidelberg, Germany \\ e-mail: flores@mpia.de; flock@mpia.de \\ 2 RIKEN Cluster for Pioneering Research, 2-1 Hirosawa, Wako-shi, Saitama 351-0198, Japan \\ e-mail: ryohei.nakatani@riken.jp
}

Received 30 August 2020 / Accepted 11 October 2020

\begin{abstract}
Context. In recent years hydrodynamical (HD) models have become important to describe the gas kinematics in protoplanetary disks, especially in combination with models of photoevaporation and/or magnetically driven winds. Our aim is to investigate how vertical shear instability (VSI) could influence the thermally driven winds on the surface of protoplanetary disks.

Aims. In this first part of the project, we focus on diagnosing the conditions of the VSI at the highest numerical resolution ever recorded, and suggest at what resolution per scale height we obtain convergence. At the same time, we want to investigate the vertical extent of VSI activity. Finally, we determine the regions where extreme UV (EUV), far-UV (FUV), and X-ray photons are dominant in the disk.

Methods. We perform global HD simulations using the PLUTO code. We adopt a global isothermal accretion disk setup, 2.5D ( 2 dimensions, 3 components) which covers a radial domain from 0.5 to 5.0 and an approximately full meridional extension. Our simulation runs cover a resolution from 12 to 203 cells per scale height.

Results. We determine 50 cells per scale height to be the lower limit to resolve the VSI. For higher resolutions, $\geq 50$ cells per scale height, we observe the convergence for the saturation level of the kinetic energy. We are also able to identify the growth of the "body" modes, with higher growth rate for higher resolution. Full energy saturation and a turbulent steady state is reached after 70 local orbits. We determine the location of the EUV heated region defined by $\Sigma_{r}=10^{19} \mathrm{~cm}^{-2}$ to be at $H_{\mathrm{R}} \sim 9.7$ and the FUV-X-ray heated boundary layer defined by $\Sigma_{r}=10^{22} \mathrm{~cm}^{-2}$ to be at $H_{\mathrm{R}} \sim 6.2$, making it necessary to introduce a hot atmosphere. For the first time we report the presence of small-scale vortices in the $r-Z$ plane between the characteristic layers of large-scale vertical velocity motions. Such vortices could lead to dust concentration, promoting grain growth. Our results highlight the importance of combining photoevaporation processes in the future high-resolution studies of turbulence and accretion processes in disks.
\end{abstract}

Key words. hydrodynamics - instabilities - methods: numerical - protoplanetary disks

\section{Introduction}

Hydrodynamical (HD) simulations in protoplanetary disks have gained substantial attention during the last decades. Due to the low coupling between the magnetic field and the gas in a large fraction of the disk (Turner et al. 2014; Dzyurkevich et al. 2013) we expect that HD instabilities play a more important role in the gas kinematics and the overall gas evolution in protoplanetary disks. In theory, the presence of vertical shear instability (VSI; Goldreich \& Schubert 1967; Fricke 1968) in purely HD accretion disks has been well studied by careful perturbational analysis and numerical simulations in several works (e.g., Nelson et al. 2013; Stoll \& Kley 2014; Barker \& Latter 2015; Lin \& Youdin 2015). Several works have shown the importance of this instability to explain the turbulence and angular momentum transport in the disk (Arlt \& Urpin 2004; Nelson et al. 2013; Stoll \& Kley 2014). The main ingredients of the VSI are a short thermal relaxation timescale and a vertical gradient of the rotational velocity in the disk. However, the effect of the VSI together with realistic thermal profiles in the disk are not well known. The evolution of the VSI has been tested in the context of planet formation and disk evolution. Recent simulations conducted by Lin (2019) have found that the VSI can be suppressed by dust settling and grain growth; however, the streaming instability (SI) may be present when VSI is active in the turbulent disk (Schäfer et al. 2020). In the context of disk evolution its been proposed that photoevaporation by energetic photons is a potential agent of disk dispersal (e.g., Shu et al. 1994; Hollenbach et al. 1994; Clarke et al. 2001; Owen et al. 2012; Alexander et al. 2006a,b, 2014; Ercolano \& Pascucci 2017). Investigating the effect of high-energy radiation on protoplanetary disks can be quite complex, but recent efforts have attempted to explain the effect of high-energy radiation onto the disk through observations of winds (e.g., Ercolano et al. 2008; Gorti et al. 2009; Owen et al. 2010, 2011). A prominent signature of photoevaporation processes is the detection of forbidden lines in the protoplanetary disk spectrum. The detection of the NeII line from the surface of TW Hya at around 10 AU (Pascucci et al. 2011), shows the important influence of high-energy photons on the surface layers of the disk; however, the vertical extension of the wind is still quite inconclusive. Moreover, Ballabio et al. (2020) found the synthetic line profile of NeII to be consistent with thermally driven winds, while 
other forbidden molecules such as OI, OII, and SII require a different scenario, possibly magneto driven winds (Fang et al. 2018; Banzatti et al. 2019). A photoevaporative process in the inner disk ( $<5$ AU) has been tested by Wang et al. (2019) and Gressel et al. (2020) that considered hydromagnetic diffusive effects, thermochemistry, and ray-tracing radiative transfer, but the wind description in terms of the Keplerian rotation and mass loss rate is different. Gressel et al. (2020) determine that the deviation of the winds caused by the magneto-centrifugal mechanism are super-Keplerian, while Wang et al. (2019) found it to be sub-Keplerian. The mass loss in Gressel et al. (2020) is $\sim 10^{-7} M_{\odot} \mathrm{yr}^{-1}$, whereas Wang et al. (2019) is $\sim 10^{-8} M_{\odot} \mathrm{yr}^{-1}$.

The coexistence between the VSI and photoevaporation processes by stellar photons is of particular interest, especially in regions where we expect a steep temperature profile and fast thermal relaxation timescales, which few have found that the VSI might operate down to $\sim 1$ AU (e.g., Klahr et al. 2018; Lyra \& Umurhan 2019; Pfeil \& Klahr 2019, 2020). The effect of viscous heating in the disk remains relatively small, making it plausible to use passive irradiated disk models to accurately represent the thermal structure (Flock et al. 2019). As a first step towards including photoevaporation processes in our HD simulations, our aim in this first paper is to study the convergence, the strength, and the region where the VSI operate in the disk, using the full meridional domain.

The structure of this paper is as follows. In Sect. 2 we present the methodology, and we describe the disk model and boundary conditions to resolve the VSI using a global 2D isothermal accretion disk configuration. In Sect. 3 we present the results with a careful analysis of the kinetic energy and vertical velocity from a global and local perspective. We also provide predictions of the influence that extreme ultraviolet (EUV), far-ultraviolet (FUV), and X-ray photons can have in the inner parts of the disk. In Sect. 4 we discuss and present a perspective of future work regarding the implications for planet formation and winds in the disk. Finally, in Sect. 5 we present our conclusions.

\section{Methods}

We performed our HD simulations using PLUTO $4.3^{1}$ by Mignone (2007). The dynamics of the fluids is described by the conservation laws accounting for the divergence of mass density and momentum density. The HD fundamental equations in our setup are

$\frac{\partial \rho}{\partial t}+\nabla \cdot(\rho \boldsymbol{v})=0$

$\frac{\partial(\rho \boldsymbol{v})}{\partial t}+\nabla \cdot(\rho \boldsymbol{v} \boldsymbol{v})+\nabla P=-\rho \nabla \Phi$

where $\rho$ is the mass density, $\boldsymbol{v}$ is the velocity vector, $\rho \boldsymbol{v}$ is the momentum density vector, and $P$ is the gas pressure. We select the isothermal equation of state with $P=c_{\mathrm{s}}^{2} \rho$, where $c_{\mathrm{s}}$ is the isothermal sound speed. The code solves consistently the HD equations in a 2D geometry and considers the system in spherical coordinates $(r, \theta, \phi)$ with axisymmetry in the azimuth. The grid cells are set up with a logarithmic increase in the radial domain and a uniform spacing in $\cos (\theta)$ for the meridional domain (e.g., Ormel et al. 2015) in order to have a better resolution around the midplane layers. The simulations are scale free. We set the

1 PLUTO 4.3 is an open source code available for download: http://plutocode.ph.unito.it/download.html
Table 1. Grid setup and initial parameters for the different runs.

\begin{tabular}{lcc}
\hline \hline$N_{\mathrm{r}} \times N_{\theta} \times N_{\phi}$ & $\Delta r[\mathrm{AU}]$ & $\Delta \theta[\mathrm{AU}]$ \\
\hline $256 \times 256 \times 1$ & $0.5: 5$ & $0.001: 3.1405$ \\
$512 \times 512 \times 1$ & $0.5: 5$ & $0.001: 3.1405$ \\
$1024 \times 1024 \times 1$ & $0.5: 5$ & $0.001: 3.1405$ \\
$2048 \times 2048 \times 1$ & $0.5: 5$ & $0.01: 3.1315$ \\
$4096 \times 4096 \times 1$ & $0.5: 5$ & $0.02: 3.1216$ \\
\hline \multicolumn{3}{c}{ Initial parameters } \\
\hline$p$ & -1.5 \\
$q$ & -1.0 \\
$R_{0}$ & 1.0 \\
$H_{0}$ & 0.1 \\
$\Sigma_{0}\left[\mathrm{~g} \mathrm{~cm}^{-2}\right]$ & 1700 \\
\hline
\end{tabular}

radial domain extending from 0.5 to 5.0 and the vertical domain covering approximately $180^{\circ}$.

\subsection{Disk model and boundary conditions}

Protoplanetary disks, rotating with Keplerian angular velocity and vertical stratification are dynamically stable according to the Solberg-Høiland criteria (e.g., Rüdiger et al. 2002). The accretion disk setup in equilibrium in cylindrical coordinates $(R, Z)$ (Nelson et al. 2013) are defined as

$$
\begin{aligned}
& \rho(R, Z)=\rho_{0}\left(\frac{R}{R_{0}}\right)^{p} \exp \left(\frac{G M}{c_{\mathrm{s}}^{2}}\left[\frac{1}{\sqrt{R^{2}+Z^{2}}}-\frac{1}{R}\right]\right), \\
& \Omega(R, Z)=\Omega_{\mathrm{k}}\left[(p+q)\left(\frac{H_{\mathrm{R}}}{R}\right)^{2}+(1+q)-\frac{q R}{\sqrt{R^{2}+Z^{2}}}\right]^{1 / 2},
\end{aligned}
$$

where $\rho_{0}=\frac{\Sigma_{0}}{\sqrt{2 \pi} H_{0} R_{0}}=4.5 \times 10^{-10} \mathrm{~g} \mathrm{~cm}^{-3}=2.7 \times 10^{14} \mathrm{~cm}^{-3}$ is the initial density at the midplane, and $\Sigma_{0}$ is the initial surface density (see Table 1 ), $R_{0}$ is the reference radius, $H_{0}$ is the reference scale height, $G$ is the gravitational constant, $M$ is the mass of the star, $p$ is the power-law fitting exponent of the density profile, and $\Omega_{\mathrm{k}}=\sqrt{G M / R^{3}}$ is the Keplerian frequency. The disk scale height in terms of the radius is

$H_{\mathrm{R}}=H_{0}\left(\frac{R}{R_{0}}\right)^{(q+3) / 2}$,

where the reference scale height ratio is $H_{0} / R_{0}=0.1$ (see Table 1) and $c_{\mathrm{s}}$ is related to the temperature through the power-law fitting exponent $q$. The radial profile of $c_{\mathrm{s}}$ is given based on Eq. (5) so that $c_{\mathrm{s}}=H_{\mathrm{R}} / \Omega_{\mathrm{k}}$. The scale length of the vertical mode is proportional to $H_{0}$.

In order to compute the fluxes in each cell, we employ second-order piece-wise linear spatial reconstruction. This reconstruction method is appropriate for both uniform and non-uniform grid spacing based on the approach presented in Mignone (2014). We use the Harten, Lax, Van Leer (HLL) Riemann solver. For the time integration we adopt a secondorder Runge-Kutta algorithm and we set the Courant-FriedrichsLewy (CFL) number to 0.25 . Table 1 summarizes the model parameter and the different runs for each resolution. For 101 and 203 cells per $H_{\mathrm{R}}$, the $\theta$ inner limit is shifted slightly inwards due to the high computational precision issue when increasing 
the resolution at the $\theta$ boundary. We investigated the third-order piecewise parabolic method (PPM3) spatial reconstruction using third-order Runge-Kutta (RK3) for the time integration for all resolution cases. We found that the PPM3 could resolve the VSI for even lower resolutions; however, our PPM3 configuration is currently is not yet stable. Therefore, we only show our analysis and discussion for the VSI using the linear reconstruction method for this paper.

\subsection{EUV, FUV, and X-ray column density}

The inner parts of the disk are highly influenced by the stellar radiation potentially ionizing the upper layers of the disk. As a first step to implement photoheating processes caused by stellar EUV, FUV, and X-ray photons, we calculate the radial column density locally to predict at what scale height the EUV and Xray-FUV heated layers are most dominant. The radial column density is calculated by integrating the hydrogen nuclei column density along the radial line of sight

$\Sigma_{r}(r)=\int_{R_{*}}^{r} n_{\rho} \mathrm{d} r$,

where $R_{*}$ is the star radius and $n_{\rho}$ is the number density of hydrogen nuclei. For this first paper in the series, since we do not consider detailed chemistry or the frequency of UV photons, we determine the boundaries in terms of $\Sigma_{r}$ by post-processing. We focus on the definition of the radial column density to locate the boundary of the ionized region (EUV heated region) and the neutral layer (X-ray-FUV heated layer) which is empirically determined from the simulations of Nakatani et al. (2018a,b). We select the boundary between the ionized or EUV heated region, namely the $\mathrm{H}-\mathrm{H}^{+}$boundary, to be located at $\Sigma_{r}=10^{19} \mathrm{~cm}^{-2}$, assuming the EUV stellar luminosity is $\sim 10^{30} \mathrm{erg} \mathrm{s}^{-1}$. The EUV heated region typically has temperatures of orders of magnitude between $10^{3}$ and $10^{4} \mathrm{~K}$ and the hydrogen is fully ionized here. The boundary of the X-ray-FUV heated layer is defined at $\Sigma_{r}=10^{22} \mathrm{~cm}^{-2}$, where the gas is heated to $10^{3} \mathrm{~K}$ and it dominates in the neutral layers. While soft X-rays are considered to be an important component for photoevaporation, the locations of FUV and X-ray heated layers are nearly identical $\Sigma_{r} \leq$ $10^{21-22} \mathrm{~cm}^{-2}$ (Gorti \& Hollenbach 2008; Nakatani et al. 2018b). Therefore, setting the boundary of the neutral photo-heated layer at $\Sigma_{r} \leq 10^{21-22} \mathrm{~cm}^{-2}$ is valid even in cases where X-rays effects are included. It should be noted that we neglect scattered light in our ray-tracing. For more information about the effects of stellar photons and the launch of winds, see Sects 4.2 and 4.3.

\section{Results}

\subsection{Dynamical evolution}

In order to investigate the kinematics we start by looking at the time evolution of the kinetic energy. Figure 1 shows the average kinetic energy at different grid cell resolutions. Our prescription of the global kinetic energy follows

$E_{\mathrm{kin}}=\frac{1}{2} \int_{v} \rho v_{Z}^{2} \mathrm{~d} V$

where $v_{Z}=v_{r} \cos (\theta)-v_{\theta} \sin (\theta)$ is the vertical component of the velocity and $E_{\text {kin }}$ is normalized with respect to

$E_{\text {norm }}=\frac{1}{2} \int_{v} \rho v_{k}^{2} \mathrm{~d} V$

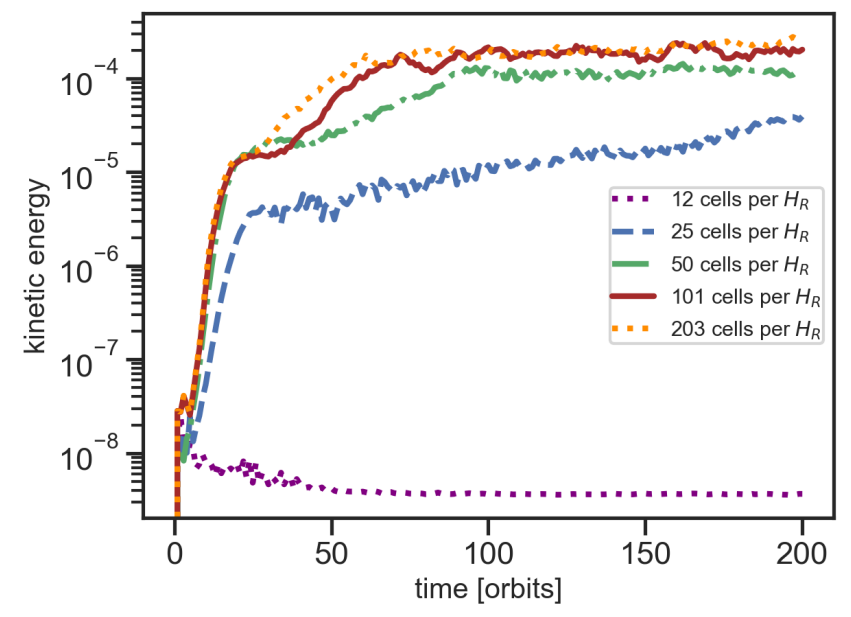

Fig. 1. Time evolution of the kinetic energy for different resolutions and space averaged at $R_{0}=1 \mathrm{AU}$. The kinetic energy is normalized with the kinetic energy from pure Keplerian velocity. The different resolutions are color-coded (see inset).

where $v_{k}$ is the Keplerian velocity. Due to our large global domain, it becomes necessary to transform our spherical velocity components to cylindrical when describing the vertical kinetic energy. For the resolution analysis we simulate a large parameter space, starting from 12 cells per scale height until 203 cells per scale height. At the lowest resolution we observed that the VSI is not resolved as the kinetic energy is not growing (see Fig. 1). We observe that the initial perturbations are damped and the kinetic energy is decreasing leading to a fully laminar disk. For resolutions higher than 12 cells per scale height, the growth seen at $\geq 25$ orbits is characteristic of the initial increase phase of the kinetic energy as the disk become unstable to the VSI, as found by Nelson et al. (2013). The 25 cells per scale height case is often used in the literature as a standard resolution to perform global simulations. However, we note that this resolution does not fully solve the VSI in accretion disks. It reaches an energy saturation slightly lower than $10^{-5}$, and after that it starts to increase monotonically without reaching a steady state, at least within the first 200 orbits. Because it is characteristic for all resolutions, the first energy saturation $\sim 10^{-5}$ is a consequence of the linear phase of the instability spreading throughout the system. The second phase of increasing kinetic energy might be related to the slower growth of the body modes that start to become visible after 25 orbits, when the quickly growing finger modes have already saturated. During this second phase, the body modes grow in the disk until they reach saturation after around 90 orbits. Our results show that at a resolution of about 100 cells per scale height the kinetic energy levels converge. For even higher resolutions, which are computationally very expensive, we predict the same convergence trend.

Our total kinetic energy follows the same trend as the perturbed kinetic energy from Nelson et al. (2013). The resolution for the VSI used by Cui \& Bai (2020) lies between 96 and 108 cells per scale height, which is in good agreement with our resolution study as well. Figure 2 shows a snapshot of the three main physical quantities in our analysis: the number density, the vertical velocity, and the kinetic energy in physical units. Overplotted are the contour lines in which the EUV heated region and the X-ray-FUV heated boundary layer are defined, based on our definition of $\Sigma_{r}$ (Sect. 2.2).

The density (Fig. 2a) has a smooth profile until it reaches the upper layers. Our density floor starts to become important 


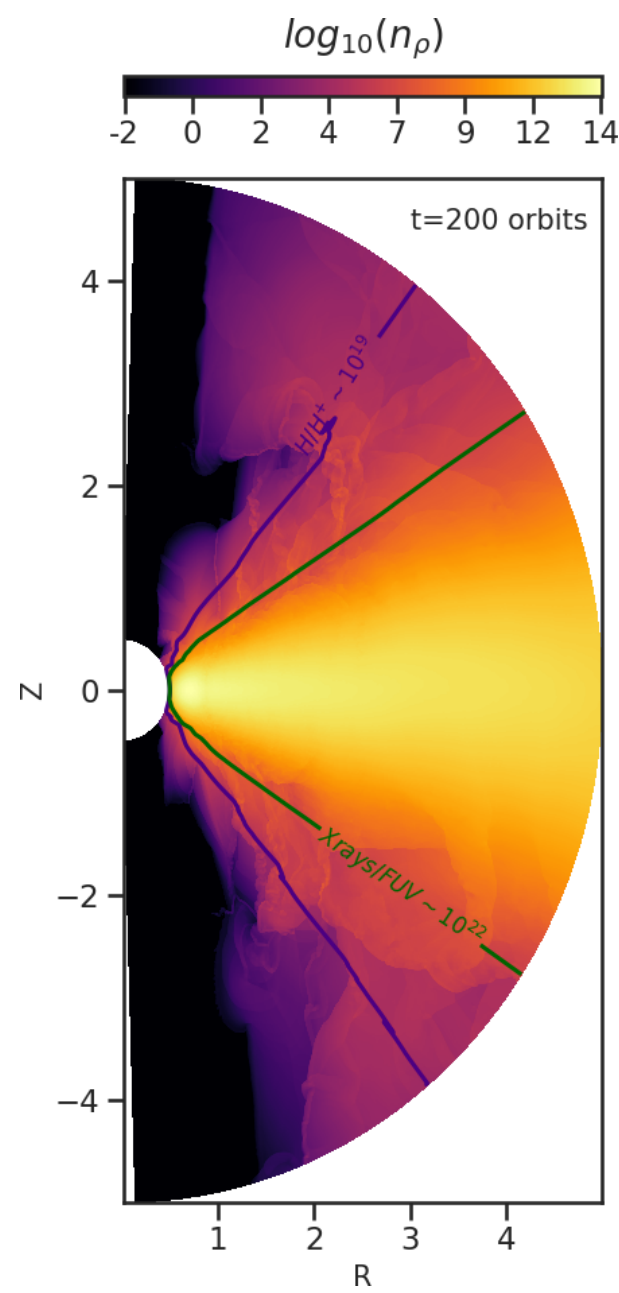

(a)
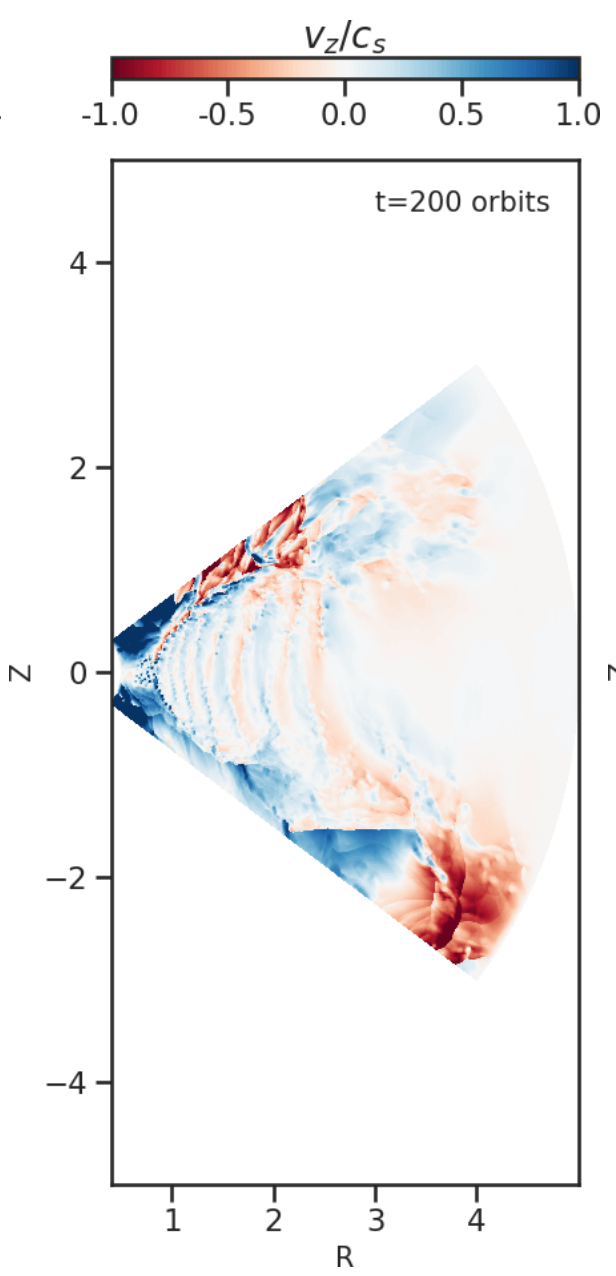

(b)
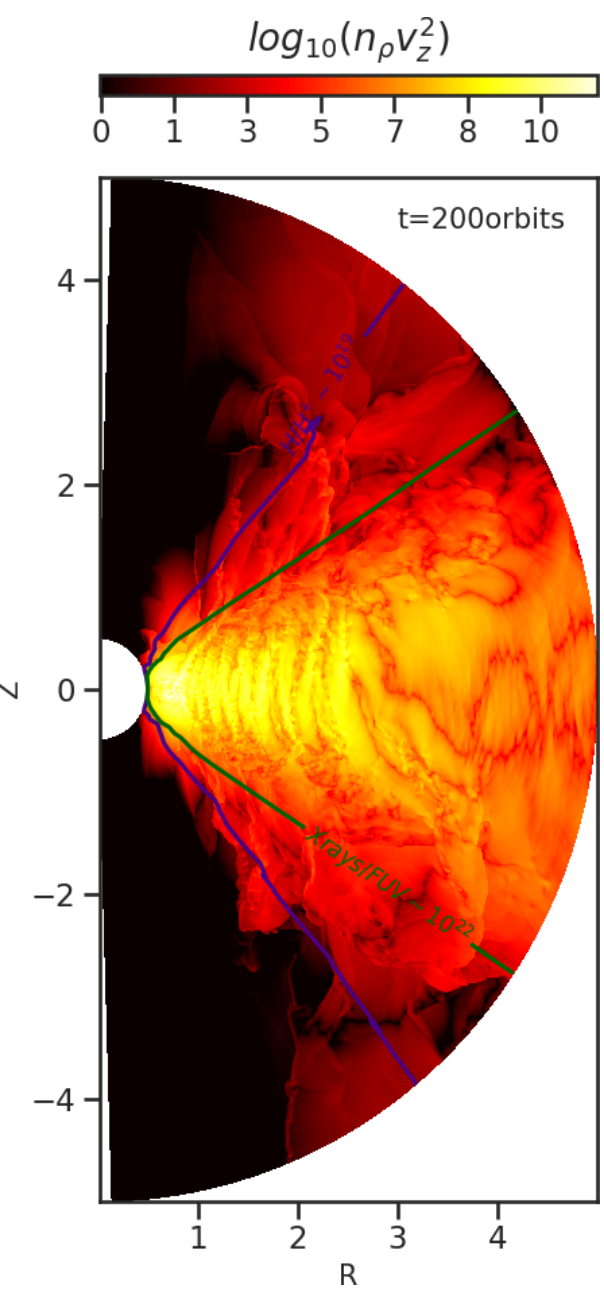

(c)

Fig. 2. Snapshot of the three main global variables in the simulation. Panel $a$ : number density in $\mathrm{cm}^{-3}$. Panel $b$ : vertical velocity normalized with respect to the sound speed. For better visibility of the midplane region, we show only an extent of $\pm 53^{\circ}$ with respect to the midplane. Panel $c$ : vertical kinetic energy in $\mathrm{g} \mathrm{cm}^{-1} \mathrm{~s}^{-2}$. Overplotted are contour solid lines where the column density of the EUV-heated region is defined by $\Sigma_{r}=10^{19} \mathrm{~cm}^{-2}$ (in purple), and the column density of the X-rays/FUV-heated boundary layer is defined by $\Sigma_{r}=10^{22} \mathrm{~cm}^{-2}$ (in dark green). The number density of hydrogen nuclei at the $\mathrm{H} / \mathrm{H}^{+}$boundary is $\sim 10^{9} \mathrm{~cm}^{-3}$ and at the X-rays/FUV-heated layer is $\sim 10^{12} \mathrm{~cm}^{-3}$.

at $H_{\mathrm{R}}=13$, which lies in a region where we do not expect VSI activity. As expected, the FUV photons penetrate deeper than EUV photons within the first 5 AU (Fig. 2). The X-rayFUV heated layer and EUV heated region reach $H_{\mathrm{R}}=6.2$ and $H_{\mathrm{R}}=9.7$, respectively. At this photoevaporation region we predict that HI regions become optically thick against EUV with a $\Sigma_{r}=10^{19} \mathrm{~cm}^{-2}$ to $\Sigma_{r}=10^{22} \mathrm{~cm}^{-2}$ (Fig. A.1).

The profile of the vertical velocity (Fig. 2b) shows clearly the upward motion shaded in blue and the downward motion shaded in red. In this region, the vertical velocity reaches a fraction of the sound speed.

The kinetic energy profile (Fig. 2c) shows the highest concentration present as corrugated wavy structures resembling the body modes in the disk. In the next section we investigate this in detail from a more local perspective.

\subsection{Local kinetic energy evolution over height and time}

We show the time-evolution of the kinetic energy over $H_{\mathrm{R}}$ at $R_{0}$ in Fig. 3. We transform the velocity vectors to cylindrical and, in order to see the local kinetic energy in a more continuous spatial structure, we then use a cubic method to interpolate the data on a new grid space with a $Z$ direction between -1 and $1 \mathrm{AU}$ at $R_{0}$. Figure 3 shows that the kinetic energy within few first orbits represents the fast growth of the finger modes in the upper layers of the disk, which is expected. At the midplane, there is no VSI activity since the vertical shear vanishes right at the midplane. Once the body modes grow, the large-scale velocity perturbations threaten the upper and lower hemisphere. At this stage the kinetic energy at the midplane also grows.

Two important features are depicted from all resolution cases in Fig. 3. The first is the presence at the beginning of vertical lanes of kinetic energy. We refer to these vertical kinetic energy lanes as finger modes, and they are consistent with the linear energy increase phases from Fig. 1. The second feature corresponds to the appearance of the body modes. As seen in Fig. 3, the growth rate of the body modes increases with resolution. For the 203 cells per scale height, the body modes saturate at around $\sim 70$ orbits. At $H_{\mathrm{R}}=0$ (midplane), the kinetic energy is very low but even more detailed analysis in the midplane is performed in the last paragraph.

Figure 4 shows the time averaged vertical profile of the kinetic energy. The vertical profile follows a trend similar to that of the density profile. Only for the lowest resolution case do we 

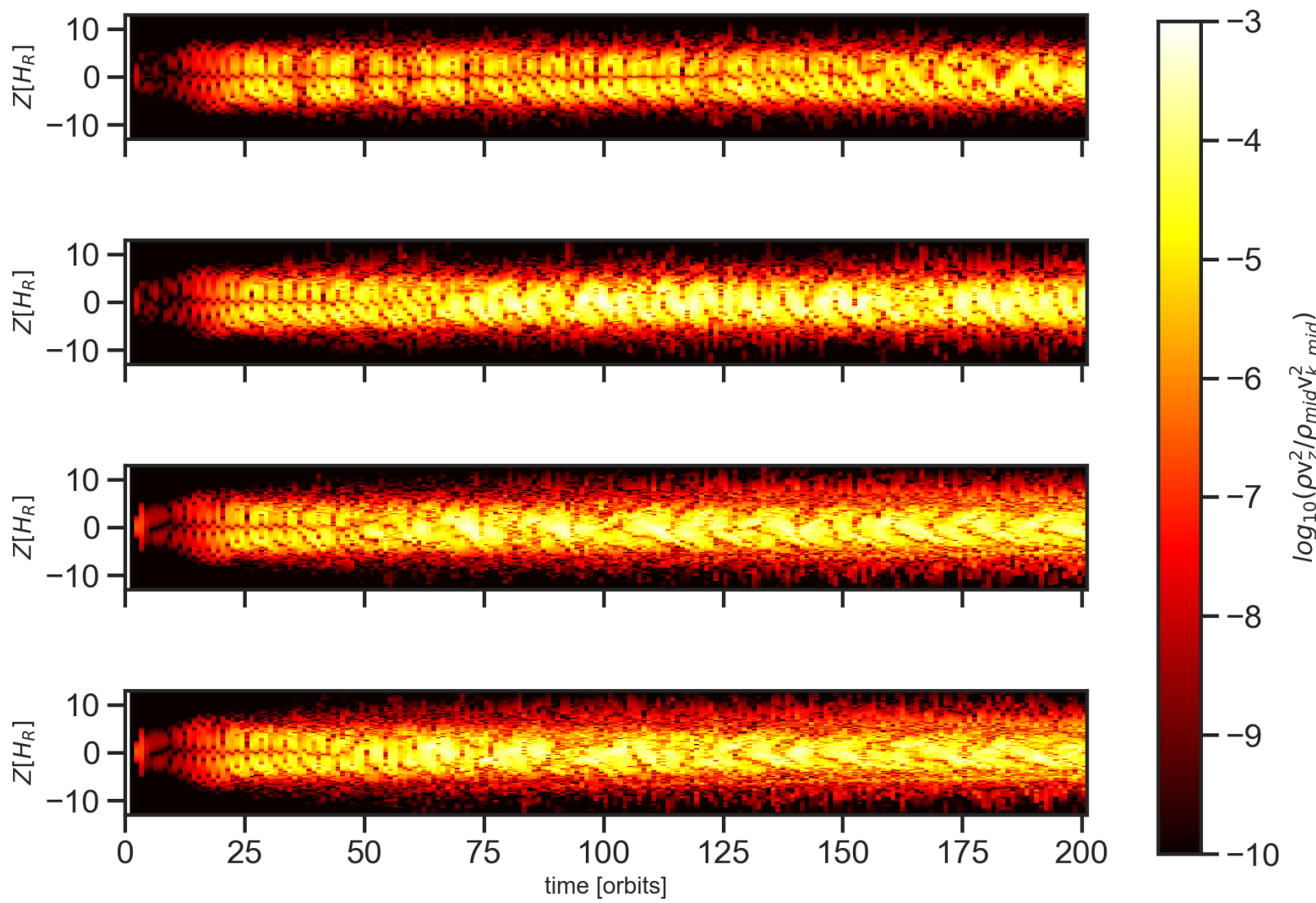

Fig. 3. Time-evolution of the local kinetic energy at $R_{0}=1$ over height for different resolutions (from top to bottom: 25, 50, 101, 203 cells per scale height). The local kinetic energy is normalized to the global kinetic energy (see Eq. (8)).

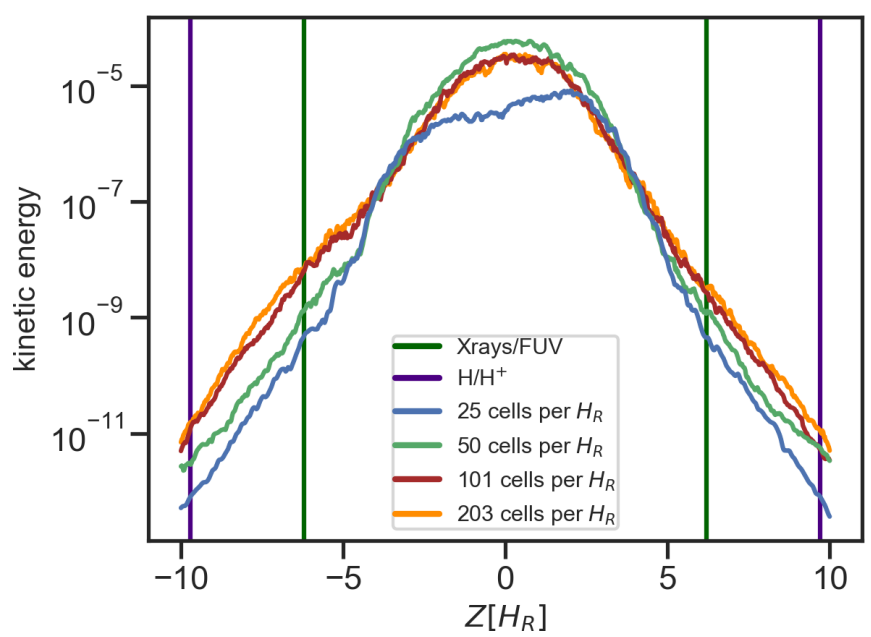

Fig. 4. Averaged profile of the local kinetic energy normalized with respect to the kinetic energy in the midplane for the $25,50,101$, and 203 cells per $H_{\mathrm{R}}$ at $R_{0}=1 \mathrm{AU}$. The vertical colored lines represent $H_{\mathrm{R}}=6.2$, where the X-ray-FUV heated boundary layer is (in dark green), and the $H_{\mathrm{R}}=9.7$, where the EUV heated region is located (in purple) (same as in Fig. 2). The models for 101 and 203 cells per $H_{\mathrm{R}}$ are in good agreement. The density floor is located $H_{\mathrm{R}}=13$, and therefore does not influence our result.

observe a plateau feature, which is due to the insufficient resolution to solve for the VSI. The VSI seems to operate in the full vertical extent. We expect a physical boundary for the VSI to be the high-temperature region caused by the FUV radiation. We also expect this position to be where the X-ray-FUV heated

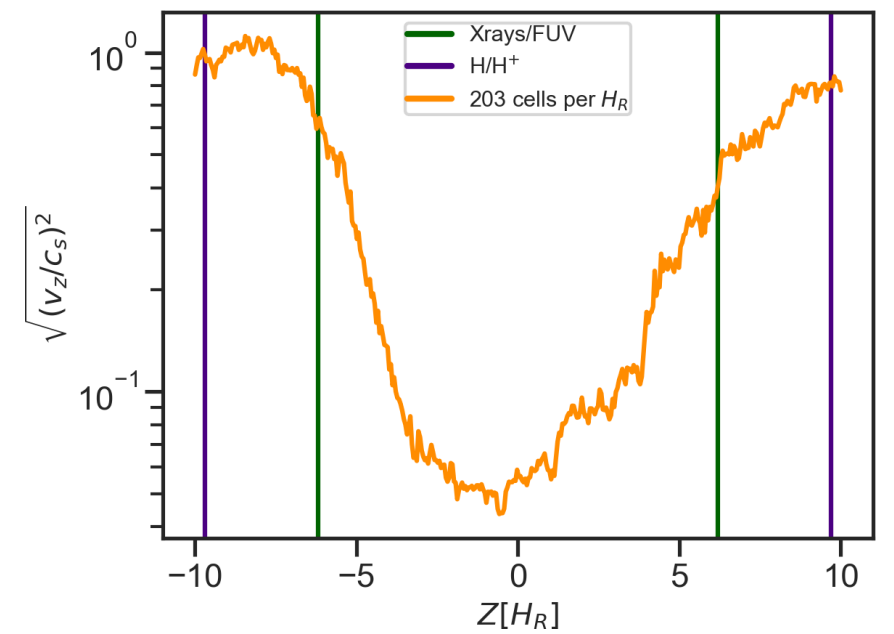

Fig. 5. Averaged profile of the vertical component of the velocity normalized with respect to the sound speed for the 203 cells per $H_{\mathrm{R}}$. The vertical colored lines are the same as in Fig. 4. At $H_{\mathrm{R}}=6.2$ the vertical velocity reaches $0.3 c_{\mathrm{s}}$. The hot ionized region is expected to start beyond $H_{\mathrm{R}}>6.2$.

boundary layer is located at $H_{\mathrm{R}}=6.2$ (see Figs. 4 and 5). Figure 5 shows that the time averaged vertical velocity increases above $60 \%$ of the sound speed at the expected wind base at $H_{\mathrm{R}}$ around 6.2. In the midplane layers the time averaged vertical motions remain small, at a level of around $1 \%$.

Figure 6 shows the time-evolution of the local kinetic energy at the midplane at $R_{0}$. The local kinetic energy at the midplane 


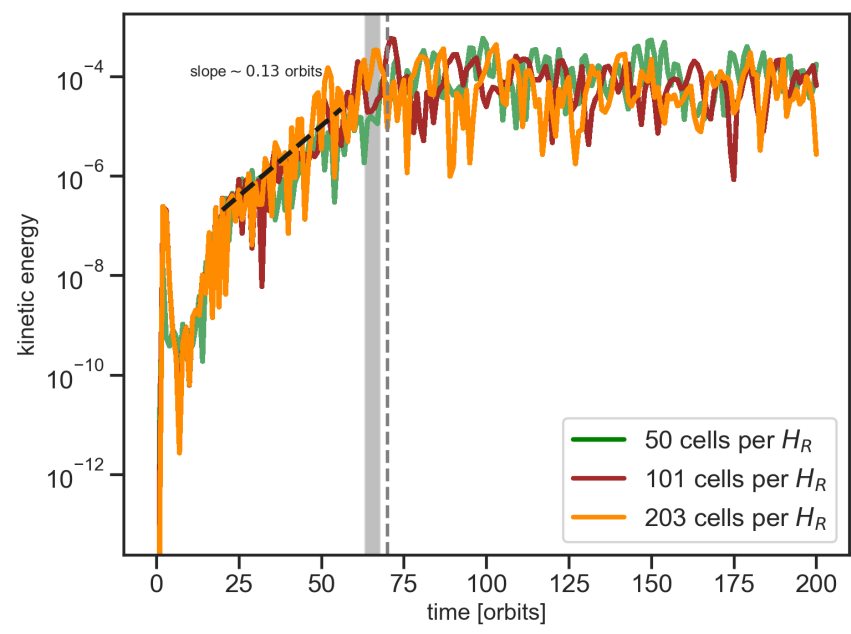

Fig. 6. Time evolution of the local kinetic energy in the midplane for the 50,101, and 203 cells per scale height resolutions at $R_{0}$. The black dashed line represent the slope of the linear growth rate phase of the local energy in the midplane. The vertical gray lane represent the approximate time where the 101 and 203 cells per scale height resolutions reach saturation and the vertical dashed line represents the time where the 50 cells per scale height converges to stability.

replicates the same two growth regimes as the averaged global kinetic energy seen in Fig. 1. The fast growth from the finger modes can even be seen at the vertical velocity at the midplane, and the slow growth of the body modes. During the growth phase of the generation of the body modes, the local kinetic energy in the midplane in all three resolutions follow a linear growth rate of $\sim 0.13$ orbits. This growth phase persists within the first $\sim 70$ orbits; it corresponds to the slow growth rate of the body modes until reaching a saturation level of $\sim 10^{-4}$ and it is 2 times slower compared to the general growth rate found by Nelson et al. (2013). The 203 and 101 cells per scale height generate the body modes faster than the 50 cells per scale height, which is the same tendency as found in Fig. 3. We conclude that we need more than 50 cells per scale height to solve the VSI in accretion disks.

\subsection{Small-scale vortices}

In Fig. 7 we show the result of the line integral convolution (LIC) method (e.g., Cabral \& Leedom 1993) to visualize the velocity magnitude vector field associated with velocity perturbations from the VSI, for the lowest and highest resolutions. The velocity magnitude includes the velocity field in every component and follows the ordinary prescription of $v_{\text {mag }}=\sqrt{v_{\mathrm{Z}}^{2}+v_{\mathrm{R}}^{2}}$, where $v_{\mathrm{R}}=v_{r} \sin (\theta)+v_{\theta} \cos (\theta)$. From the LIC method, we find small-scale vortices in both resolution cases; however, these small-scale vortices are not featured in the lowest resolution case.

The possibility of the presence of vortices formed by VSI was first found by Richard et al. (2016) in the $r-\phi$ plane. These vortices can be long-lived locally around 500 orbits (Manger \& Klahr 2018) with an $H_{\mathrm{R}}=0.1$ and with an azimuthal and radial extension of about 40 and 4 au (Flock et al. 2020), respectively. In our case the vortices appear in the $r-Z$ plane, mostly inside the region between two large-scale upward and downward motions. They might be related to Kelvin-Helmholtz instabilities appearing between these large-scale motions, as was described by Latter \& Papaloizou (2018). Moreover, these small-scale vortices happen in an $H_{\mathrm{R}}<5$, and are therefore more important for dust evolution.

\section{Implications for planet formation and winds in disk}

The VSI is a large-scale global instability that potentially plays an important role in the evolution and dynamics of protoplanetary disks. In the midplane the dust settling overcomes the VSI, and as the dust concentrates and grows to millimeter size and greater, these particles settle against VSI and eventually undergo SI to favor planet formation (Lin 2019). However, the VSI in combination with the SI leads to radial dust concentration in long-lived accumulations which are significantly denser areas than those formed by the SI alone (Schäfer et al. 2020).

We observe symmetric features, which have not been observed before, after 100 orbits in the time evolution of the kinetic energy (Fig. 3). Furthermore, we refer to the LIC plots (Fig. 7) that show the presence of small vortices along the midplane, where grains can be concentrated. We encourage further analysis of this manner and its effect on planet formation in the future.

\subsection{Importance of stellar photon layers and the launch of winds}

The VSI can maintain small particles $(<100 \mu \mathrm{m})$ floating (Lin 2019) until they eventually become ionized by FUV photons at the disk surface and contribute to heating in the atmosphere. Both the photoionization effects and the high temperature in this region make the surface of the disk and atmosphere an optimal scenario for photoevaporation processes, resulting in the launch of winds. In the disk atmosphere the MHD winds are likely favorable (Cui \& Bai 2020) at less than 5 AU. Cui \& Bai (2020) showed that VSI and MHD winds can coexist, while magnetized winds being the dominant process to transport angular momentum. However, this would depend on (1) the so-called wind base, which depends mainly on the position of the EUV and FUV layers, and (2) a realistic thermal profile. By considering the initial flux and the extinction along the radial line of sight we can determine the location and extension of the wind in the future work. The detailed role of the VSI in the mechanism of wind launching has to be investigated, especially because the magnetic coupling to the gas remains low in the FUV shielded region $\left(H_{\mathrm{R}}<6.2\right)$. Recent improvements by Wang et al. (2019) and Gressel et al. (2020), aimed at including more realistic scenarios, have included thermochemistry and irradiation in their global magnetohydrodynamical simulations.

\subsection{Frequency-integrated cross sections}

Perhaps the most robust way to define the influence of EUV and X-ray-FUV photon layers is by taking into consideration the frequency-dependent cross sections for each photon. The radial optical depth of each photon depends on the frequency range that is used in the integration of the ionization rates and the associated heating rates. This is very important to consider when characterizing the ionized region in the disk atmosphere, which is influenced by EUV photons. The atomic hydrogen abundance in this ionized region is negligible in the ionization front where we expect a radial optical depth of the EUV equal to 1 (Hollenbach et al. 1994; Tanaka et al. 2013; Nakatani et al. 2018a,b). This boundary between the ionized region and the neutral layer is instead located at an atomic hydrogen column density of $10^{18} \mathrm{~cm}^{-2}$. This atomic hydrogen column density can be translated into a hydrogen column density of $10^{19-20} \mathrm{~cm}^{-2}$ depending on the stellar EUV luminosity, and therefore is used here to define the EUV heated region. 


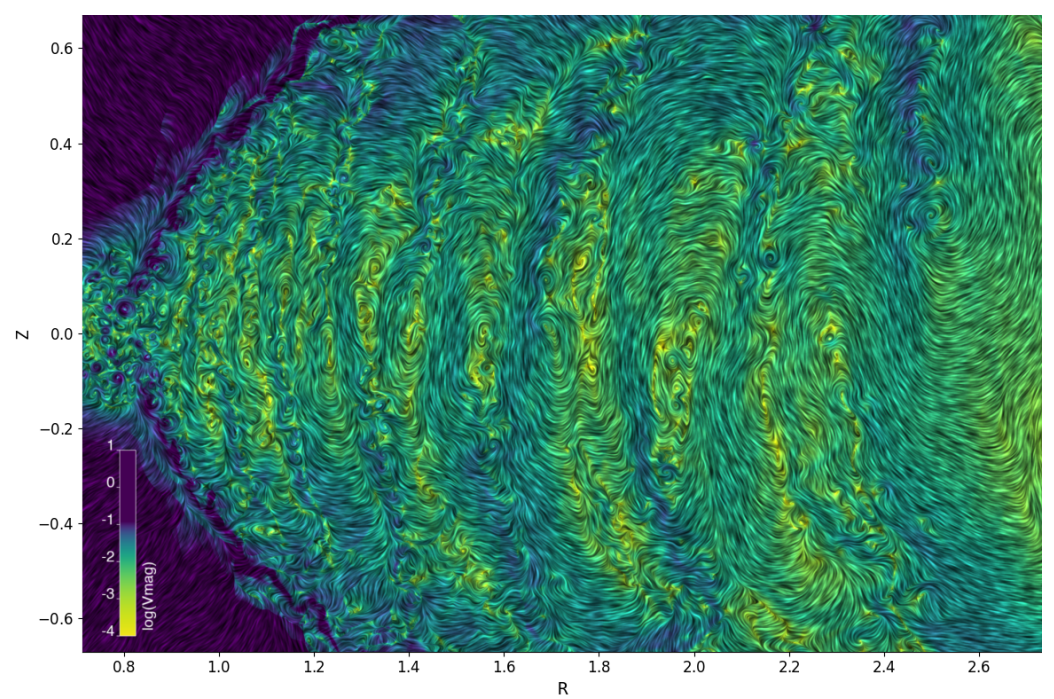

(a)

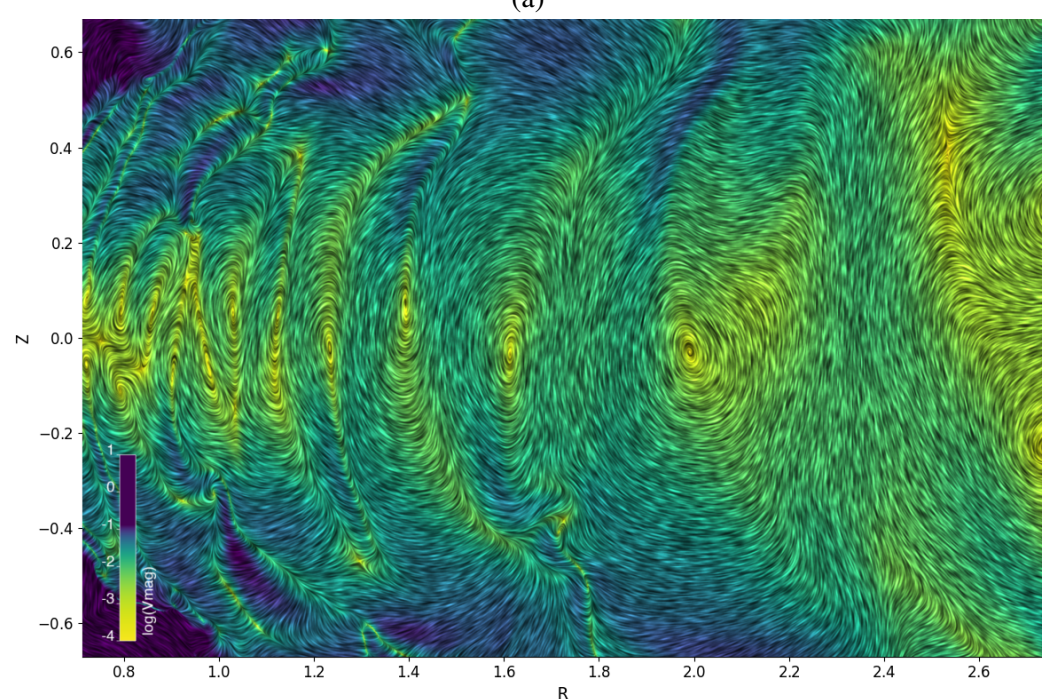

(b)

Fig. 7. Snapshot of $v_{\mathrm{mag}}$ for the 203 cells per scale height (panel a) compared to the lowest resolution of 25 cells per scale height (panel $b$ ) at 200 orbits. The colored contour plot shows $v_{\text {mag }}$ and the streamlines show the velocity flow pattern when applying the LIC method. The $v_{\text {mag }}$ range (from 0 to 0.2 in code units) is narrowed down to highlight the low-velocity perturbations close to the midplane associated with the VSI.

Given that FUV photons can promote heating via photoelectric heating (Bakes \& Tielens 1994) in the disk atmosphere, these photons are attenuated by dust once the hydrogen column density is between $10^{21}$ and $10^{22} \mathrm{~cm}^{-2}$ (Gorti et al. 2009; Nakatani et al. 2018a). When considering the radial optical depth of the FUV, the FUV opacity evaluated for graphite is about $\sim 10^{4} \mathrm{~cm}^{2} \mathrm{~g}^{-1}$, which is in the wavelength regime between 0.2 and $0.09 \mu \mathrm{m}$ $(6 \mathrm{eV}<h v<13.6 \mathrm{eV})$. Assuming a dust-to-gas mass ratio of 0.01 , this gives a total FUV opacity of $100 \mathrm{~cm}^{2} \mathrm{~g}^{-1}$. While it is more likely that the dust-to-gas mass ratio is smaller than 0.01 in the upper layers, perhaps a more nominal range could be somewhat from 0.001 to 0.0001 , shifting the radial optical depth of the FUV equal to 1 closer to the midplane. This FUV opacity prescription is consistent with the dust population used by Flock et al. (2016) to describe the physical properties of the inner rim. In realistic disk scenarios, which we consider for the second part of this project, the EUV and FUV flux relies on the wavelengthdependent initial flux we assume and the extinction along the radial line of sight at each location in the disk. Meanwhile, we expect the EUV to provide an important effect on the extension of the wind in the disk atmosphere.

\subsection{Electron density}

The temperature and electron number density can be inferred from thermally excited forbidden lines (e.g., [OI] 5577/6300 line ratio) in regions where high temperatures $(>5000 \mathrm{~K})$ lead to collisional excitations of electrons (Simon et al. 2016). Including or not the effects of X-rays, the electron relative abundance between the EUV heated layer and the X-ray-FUV heated layer is still $\sim 10^{-4}$, which comes from atomic carbon ionization by FUV. The electron density in this region can be as high as $\sim 10^{4}-10^{5} \mathrm{~cm}^{-3}$ at $\sim 1$ AU (Nakatani et al. 2018a,b). We expect that at $<1 \mathrm{AU}$, the $\mathrm{X}$-ray, EUV, and FUV fluxes are higher; therefore, photons penetrate deeper in the disk and the electron density can be higher. On the other hand, farther from the star the electron abundance decreases, and as does the electron density.

\section{Conclusions}

We performed 2.5D (axisymmetric) hydrodynamical simulations for an isothermal accretion disk with different resolutions, up to 203 cells per scale height to fully resolve for the VSI. Resolution study for the VSI is important to assess its effect in different 
regions of the disk. We determine that the standard resolution of 50 cells per scale height is the lower limit to resolve the VSI. This determination is based on the total kinetic energy saturation level. Our key points can be summarized as follows:

1. We found the VSI to operate throughout the entire disk until reaching our density floor $0.003 \mathrm{~cm}^{-3}$ at around $H_{\mathrm{R}}=13$ at $R_{0}=1$ for resolutions $\geq 50$ cells per scale height. We expect turbulent scenarios where the X-ray-FUV heated boundary layer at $H_{\mathrm{R}}=6.2$ is located, whereas the EUV heated region reaches $H_{\mathrm{R}}=9.7$. Nevertheless, we encourage the use of high resolution ( $\geq 50$ cells per scale height) to properly conduct the analysis of VSI.

2. We found at a resolution of 25 cells per $H_{\mathrm{R}}$ the appearance of a plateau around the midplane when looking at the local kinetic energy over height. This could serve as a diagnostic to check whether or not the VSI is fully resolved.

3. We observe a clear dependence on the growth rate of the body modes with resolution. For the highest resolution the growth of the body modes saturates after 70 orbits.

4. We diagnose the presence of small-scale vortices between the large-scale motion of the VSI when performing the LIC method. These small-scale vortices reach $H_{\mathrm{R}}<5.0$; therefore they are more important for dust evolution. The vertical kinetic energy shows a more symmetric oscillation for the upper and lower hemisphere for the highest resolution. In the future we will fully investigate the dynamical influence of the heated layers on the VSI and the disk evolution. A resolution of 50 cells per scale $H_{\mathrm{R}}$ seems to capture the main characteristics of the VSI, and so is suitable for future studies.

Acknowledgements. This work is supported by the European Research Council (ERC) project under the European Union's Horizon 2020 research and innovation programme number 757957. The LIC images were generated by Thomas Müller from the Haus dier Astronmomie. We would like to thank the matplotlib team (Hunter 2007) for the good quality tool in order to better visualize the data.

\section{References}

Alexander, R. D., Clarke, C. J., \& Pringle, J. E. 2006a, MNRAS, 369, 216 Alexander, R. D., Clarke, C. J., \& Pringle, J. E. 2006b, MNRAS, 369, 229

Alexander, R., Pascucci, I., Andrews, S., Armitage, P., \& Cieza, L. 2014, in Protostars and Planets VI, eds. H. Beuther, R. S. Klessen, C. P. Dullemond, \& T. Henning (Tucson, AZ: University of Arizona Press), 475

Arlt, R., \& Urpin, V. 2004, A\&A, 426, 755

Bakes, E. L. O., \& Tielens, A. G. G. M. 1994, ApJ, 427, 822

Ballabio, G., Alexander, R. D., \& Clarke, C. J. 2020, MNRAS, 496, 2932

Banzatti, A., Pascucci, I., Edwards, S., et al. 2019, ApJ, 870, 76

Barker, A. J., \& Latter, H. N. 2015, MNRAS, 450, 21

Cabral, B., \& Leedom, L. C. 1993, in Proceedings of the 20th Annual Conference on Computer Graphics and Interactive Techniques, SIGGRAPH '93 (New York, NY, USA: Association for Computing Machinery), 263

Clarke, C. J., Gendrin, A., \& Sotomayor, M. 2001, MNRAS, 328, 485

Cui, C., \& Bai, X.-N. 2020, ApJ, 891, 30

Dzyurkevich, N., Turner, N. J., Henning, T., \& Kley, W. 2013, ApJ, 765, 114

Ercolano, B., \& Pascucci, I. 2017, R. Soc. Open Sci., 4, 170114

Ercolano, B., Drake, J. J., Raymond, J. C., \& Clarke, C. C. 2008, ApJ, 688, 398

Fang, M., Pascucci, I., Edwards, S., et al. 2018, ApJ, 868, 28

Flock, M., Fromang, S., Turner, N. J., \& Benisty, M. 2016, ApJ, 827, 144

Flock, M., Turner, N. J., Mulders, G. D., et al. 2019, A\&A, 630, A147

Flock, M., Turner, N. J., Nelson, R. P., et al. 2020, ApJ, 897, 155

Fricke, K. 1968, Z. Astrophys., 68, 317

Goldreich, P., \& Schubert, G. 1967, ApJ, 150, 571

Gorti, U., \& Hollenbach, D. 2008, ApJ, 683, 287

Gorti, U., Dullemond, C. P., \& Hollenbach, D. 2009, ApJ, 705, 1237

Gressel, O., Ramsey, J. P., Brinch, C., et al. 2020, ApJ, 896, 126

Hollenbach, D., Johnstone, D., Lizano, S., \& Shu, F. 1994, ApJ, 428, 654

Hunter, J. D. 2007, Comput. Sci. Eng., 9, 90

Klahr, H., Pfeil, T., \& Schreiber, A. 2018, Handbook of Exoplanets (Cham: Springer), 138

Latter, H., \& Papaloizou, J. 2018, MNRAS, 474, 3110
Lin, M.-K. 2019, MNRAS, 485, 5221

Lin, M.-K., \& Youdin, A. N. 2015, ApJ, 811, 17

Lyra, W., \& Umurhan, O. M. 2019, PASP, 131, 072001

Manger, N., \& Klahr, H. 2018, MNRAS, 480, 2125

Mignone, A. 2007, ApJ, 170, 228

Mignone, A. 2014, Comput. Phys., 270, 784

Nakatani, R., Hosokawa, T., Yoshida, N., Nomura, H., \& Kuiper, R. 2018a, ApJ, 857,57

Nakatani, R., Hosokawa, T., Yoshida, N., Nomura, H., \& Kuiper, R. 2018b, ApJ, 865,75

Nelson, R. P., Gressel, O., \& Umurhan, O. M. 2013, A\&A, 435, 2610

Ormel, C. W., Shi, J.-M., \& Kuiper, R. 2015, MNRAS, 447, 3512

Owen, J. E., Ercolano, B., Clarke, C. J., \& Alexander, R. D. 2010, MNRAS, 401, 1415

Owen, J. E., Ercolano, B., \& Clarke, C. J. 2011, MNRAS, 412, 13

Owen, J. E., Clarke, C. J., \& Ercolano, B. 2012, MNRAS, 422, 1880

Pascucci, I., Sterzik, M., Alexander, R. D., et al. 2011, ApJ, 736, 13

Pfeil, T., \& Klahr, H. 2019, ApJ, 871, 150

Pfeil, T., \& Klahr, H. 2020, ApJ, submitted [arXiv:2008 . 11195]

Richard, S., Nelson, R. P., \& Umurhan, O. M. 2016, MNRAS, 456, 3571

Rüdiger, G., Arlt, R., \& Shalybkov, D. 2002, A\&A, 391, 781

Schäfer, U., Johansen, A., \& Banerjee, R. 2020, A\&A, 635, A190

Shu, F., Najita, J., Ostriker, E., et al. 1994, ApJ, 429, 781

Simon, M. N., Pascucci, I., Edwards, S., et al. 2016, ApJ, 831, 169

Stoll, M. H. R., \& Kley, W. 2014, A\&A, 572, A77

Tanaka, K. E. I., Nakamoto, T., \& Omukai, K. 2013, ApJ, 773, 155

Turner, N., Fromang, S., Gammie, C., et al. 2014, in Protostars and Planets VI, eds. H. Beuther, R. Klessen, C. Dullemond, \& T. Henning, (Tucson, AZ: University of Arizona Press), 411

Wang, L., Bai, X.-N., \& Goodman, J. 2019, ApJ, 874, 90

\section{Appendix A: Radial column density}

We conduct a surface density analysis for the determination of the EUV heated region and X-ray-FUV heated boundary layer (see Sect. 2.2). Figure A.1 shows the contour plot of the radial column density; overplotted are the two regions of interest.

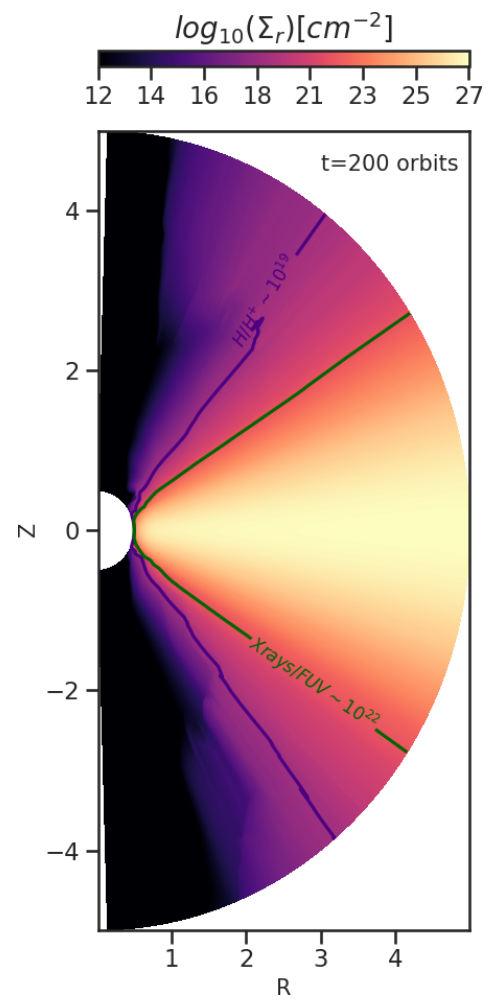

Fig. A.1. Radial column density in $\mathrm{cm}^{-2}$. Overplotted are solid lines where $\Sigma_{r}=10^{19} \mathrm{~cm}^{-2}$ represents the EUV heated region (in purple), and $\Sigma_{r}=10^{22} \mathrm{~cm}^{-2}$ represents the X-ray-FUV heated boundary layer (in dark green). 